\title{
Controlled Sub-Micrometer Hierarchical Textures Engineered In Polymeric Fibers And Micro-Channels Via Thermal Drawing
}

Tung Nguyen-Dang, Alba C. de Luca, Wei Yan, Yunpeng Qu, Alexis G. Page, Marco Volpi, Tapajyoti Das Gupta, Stéphanie P. Lacour, and Fabien Sorin*

*Corresponding author: Fabien.Sorin@epfl.ch

T. Nguyen-Dang, W. Yan, Y. Qu, A. Page, M. Volpi, T. Dasgupta and Prof. F. Sorin, Institute of Materials, École Polytechnique Fédérale de Lausanne (EPFL), 1015 Lausanne (Switzerland).

A. C. de Luca and Prof. S. P. Lacour

Bertarelli Foundation Chair in Neuroprosthetic Technology, Laboratory for Soft Bioelectronic Interfaces, Institute of Microengineering, Institute of Bioengineering, Centre for Neuroprosthetics, École Polytechnique Fédérale de Lausanne (EPFL), 1015 Lausanne (Switzerland).

Keywords: multi-material fibers; micro and nano fabrication; patterning of high curvature surfaces; large-area functionalized surfaces; smart textiles; bio-materials interfaces.

\begin{abstract}
:
The controlled texturing of surfaces at the micro- and nano-scales is a powerful method for tailoring how materials interact with liquids, electromagnetic waves, or biological tissues. The increasing scientific and technological interest in advanced fibers and fabrics has triggered a strong motivation for leveraging the use of textures on fiber surfaces. Thus far however, fiberprocessing techniques have exhibited an inherent limitation due to the smoothing out of surface textures by polymer reflow, restricting achievable feature sizes. In this article, we establish a theoretical framework from which we develop a strategy to reduce the surface tension of the textured polymer, thus drastically slowing down thermal reflow. We demonstrate with this approach the fabrication of potentially kilometers-long polymer fibers with controlled hierarchical surface textures of unprecedented complexity and with feature sizes down to a few hundreds of nanometers, two orders of magnitude below current configurations. Using such fibers as molds, we also fabricate 3D micro-channels with textured inner surfaces within soft polymers such as PDMS, at dimensions and a degree of simplicity impossible to reach with current techniques. This strategy for the texturing of high curvature surfaces opens novel opportunities in bioengineering, regenerative scaffolds, microfluidics, and smart textiles.
\end{abstract}




\section{Introduction}

The development of kilometers-long multi-material fibers exhibiting increasingly complex functionalities is opening unforeseen opportunities for flexible electronics and optoelectronics, health care via optical probes or advanced scaffolds, sensing and monitoring, energy harvesting and smart textiles ${ }^{[1-4]}$. Novel techniques have emerged to functionalize the tip of optical fibers ${ }^{[5-}$ ${ }^{7]}$, or depositing materials onto ${ }^{[8]}$ or within the pores ${ }^{[9-13]}$ of microstructured fibers. Such multifunctional fibers are envisioned as a novel generation of probes in imaging and endoscopic systems, in photonic interconnects, flexible logic systems or advanced opto- and micro-fluidic configurations. Recent breakthroughs in the thermal drawing process - the same technique used to fabricate conventional optical fibers - have also enabled to integrate complex materials architectures and functionalities not only locally but along the entire fiber length of tens of kilometers ${ }^{1-3}$. The thermal drawing process consists in heating above the glass transition temperature and pulling at relatively high viscosity (typically $10^{4}$ to $10^{7} \mathrm{~Pa} . \mathrm{s}$ ) a macroscopic preform made out of a glass, typically silica, or a thermoplastic. Pulling a fiber at such a high viscosity compared to solution-based processes has the key advantage of enabling a control over the interplay between the viscosity, internal stresses and surface tension. Fibers with complex cross-sectional architectures such as microstructured photonic crystal fibers ${ }^{[14,15]}$ can be fabricated with this approach, at the simplicity and scalability associated with fiber processing. More recently, this technique was shown to also enable the fabrication of multi-material fibers that integrate polymers or glasses but also metals, inorganic semiconductors, or nanocomposites, uniformly integrated in prescribed positions along the fiber length ${ }^{1-3}$. Such advanced multi-material fiber systems have been proposed for applications, in optics ${ }^{[16-19]}$ and imaging ${ }^{[20-}$

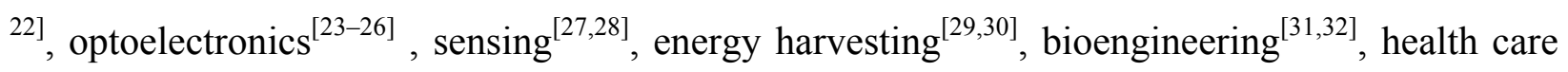
or smart textiles ${ }^{[21,33,34]}$. 
So far however, the use of micro- and sub-micrometer surface textures to impart fibers with novel functionalities has not been exploited. The texturing of materials is used in a myriad of applications to tailor surface properties and provide novel or improved functionalities. In particular, textures can be used to control optical properties ${ }^{[35]}$, to tailor how liquid spread on surfaces or flow within channels ${ }^{[36,37]}$, and to influence how cells grow and nerves regenerate ${ }^{[38-}$ 40]. The fabrication of fibers with sub-micrometer textures that could exhibit such advanced capabilities has however been to date unsuccessful because of inherent limitations associated with the thermal drawing process, and other fiber-making approaches in general. Any texture can be simply fabricated at the preform level (sub-millimeter scale), or imprinted onto a die for extrusion, but during the fiber pulling step a reflow of the desired pattern driven by surface tension (Laplace pressure) occurs ${ }^{[41,42]}$, resulting in the distortion of the initial shape at the fiber level below a certain feature size. Earlier work ${ }^{[43,44]}$ has achieved the functionalization of thermally drawn fibers that integrated textures but with large sizes, typically around 30 microns, using polyetherimide (PEI), a thermoplastic with a very high mechanical strength that allows for a processing at high viscosity that can limit reflow at these dimensions. For many applications in optics, optoelectronics, microfluidics, biology or advanced textiles however, it is necessary to have smaller - sub-micrometer - patterns on the surface and within a variety of polymers, which display much weaker mechanical strengths such as polycarbonate (PC), poly(methyl methacrylate) (PMMA) or even soft elastomers such as the widely used poly(dimethylsiloxane) (PDMS).

In this contribution, we demonstrate the fabrication of complex textures on the surface or within hollow channels of polymer fibers, with feature sizes down to a few hundreds of nanometers, using the thermal drawing technique. We exploit the thermal drawing of multi-material preforms 
by integrating a sacrificial material co-drawn in intimate contact with the desired textured polymer. This enables significant reduction of the interfacial tension hence the driving force behind the thermal reflow, leaving arbitrary textures intact down to the nanometer scale. In what follows we first describe our fabrication procedure, establish our theoretical framework to explain the observed surface textures, and demonstrate a variety of pattern configurations that can be realized. We also show that textured fibers can be used as molds for soft polymers such as PDMS to produce 3D cavities with microscopic inner patterns. These results hence also pave a simple and scalable way towards the controlled patterning of high curvature surfaces, a challenge that still resists conventional lithography approaches. To illustrate this achievement, we show how textured fibers and micro-channels can be assembled into highly functional large-area surfaces with tailored hydrophobic or optical properties, or serve as substrates to favor the alignment and growth of biological cells. We finally discuss how this technique opens intriguing opportunities in novel biological probes, opto- and microfluidics systems, advanced regenerative scaffolds and smart and biological textiles.

\section{Results}

Fabrication and modeling of textured fibers

In order to achieve micron or sub-micron feature sizes at the fiber level, it is first important to create an initial pattern of suitable size at the preform level. Commercially acquired rectangular plates of polycarbonate (PC) or poly(methyl methacrylate) (PMMA) were cut into rectangular flat preforms of typically $200 \mathrm{~mm}$ in length (L), $10 \mathrm{~mm}$ in height $(\mathrm{H})$ and $20 \mathrm{~mm}$ in width (W). These preforms, shown schematically in Figure 1(a), were then patterned with a periodic structure of square grooves with a depth $h$, a width $w$ and a periodicity $\lambda$. We chose to focus on square-shaped patterns in this study because they are simple to fabricate at the preform level, and 
since sharp features can disappear rapidly and hence allow for an uncompromising assessment of our approach. We used either milling for the large features with a periodicity above 100 micrometers, or soft embossing with a PDMS mold replicated from a Silicon master mask for smaller feature sizes (see the methods section). Note that this represents the first combination of soft lithography and thermal drawing techniques to realize functional systems with high scalability. The patterned preforms were then thermally drawn into hundreds-of-meter long fibers in a custom-made draw tower, so that the sub-millimeter texture ideally rescales to micron or even submicron feature sizes in the fiber cross-section, as shown schematically in Figure 1(a). Inevitably however, thermal reflow will occur driven by the surface tension of the polymer and the curvature of the texture ${ }^{[41,42]}$. In the top part of Figure 1(b) we show the SEM micrographs of the surface texture of two fibers with a rectangular cross-section (referred to as ribbons) made out of PC, thermally drawn with a draw down ratio (ratio of the preform to fiber heights) of 20. At the preform level, the square texture fabricated had periodicities of 200 and 40 micrometers, targeting textures of 10 and 2 micrometers at the fiber level, respectively. For these targeted sizes however, it can be seen that while the periodicity of the textures corresponds to the expected value, the $10 \mu \mathrm{m}$ structure has fainted and the $2 \mu \mathrm{m}$ one has almost entirely collapsed.

To account for this limitation, a simple approach to model the underlying flowing mechanism is used. The deformation of the structure during a preform-to-fiber drawing experiment can be decoupled into two mechanisms: the scaling deformation due to the drawing process, and the thermal deformation, or reflow, of the microstructure. The scaling deformation is related to an elongation in the drawing direction, the $z$-direction, and the thermal "reflow" relates to a flow solely in the $x y$-plane, perpendicular to the draw direction. Both mechanisms induce a velocity field that contributes independently to the size reduction of a texture's height (see Figure 1(a) 
and SI 1). Let us then consider a structure of height $h$ at a position $z$ along the drawing axis (Figure 1 and S1). At position $z+d z$, the structure has now a height $h+d h$ with $d h<0$, and $d h$ $=d h_{s c}+d h_{r e}$, where $d h_{s c}$ and $d h_{r e}$ are the contribution of scaling deformation and of thermal deformation respectively. The scaling deformation at a given cross-section $z$ is calculated from mass conservation, by assuming that the polymer is incompressible (see SI 1):

$$
d h_{s c}=-\frac{1}{2} \frac{h}{v} d v
$$

Where $h=h(z)$ and $v=v(z)$ are the local height of the structure and the local speed along the drawing direction $(z)$ of the fiber respectively. The thermal deformation, or "reflow", has been studied in previous work using different formalisms and in various configurations ${ }^{[41,42]}$. Here, in order to obtain the expression of the reflow deformation, we solve the Navier-Stokes equation for the polymer flow during the preform-to-fiber drawing process, by considering it as a Newtonian fluid in a low Reynolds number regime (see SI 1). The deformation due to thermal reflow, assuming, without loss of generality, that the square shaped periodic pattern has a simple sinusoidal shape with only one harmonic, is given by:

$$
d h_{r e}=-\frac{\pi \gamma h}{\eta \lambda} d t=-\frac{\pi \gamma h}{\eta \lambda} \frac{d z}{v}
$$

Note that in this configuration, only the height of the texture is changed but not its periodicity that is only subjected to the scaling effect. If now we consider a preform-to-fiber process with a furnace of length $L$ as shown in Figure S1, with a structure at the preform level of height $h_{0}$ at the entrance of the furnace $z=0$, the height of the structure at the output of the furnace where the final fiber is formed and the drawing speed is $v_{f}$ becomes:

$$
\left.h(L)=h_{0}\left(\frac{v_{f}}{V_{0}}\right)^{-\frac{1}{2}} \exp \left(\int_{0}^{L}-\pi \frac{\gamma}{\eta \lambda} \frac{1}{v} d z\right)\right)
$$


Despite the simplifications made in this model, equation (3) provides intriguing insights in the deformation of the structure during the thermal drawing process. The first factor corresponds to the drawn-down-ratio, $f_{s c}=\left(\frac{v_{f}}{v_{0}}\right)^{1 / 2}$, associated with any thermal drawing, that results from mass conservation as the preform is pulled into a fiber. The second factor represented by the exponential term accounts for the contribution of the Laplace pressure induced thermal reflow during drawing. Defining the reflow factor by $f_{r e}=1-\exp \left(-\int_{0}^{L} \pi \frac{\gamma}{\eta \lambda} \frac{1}{v} d z\right), f_{r e}$ varies from a value of 0 , corresponding to the scenario where the structure is perfectly conserved (no reflow), to 1 for which the structure has completely collapsed. It is worth noting upfront that the reflow factor $f_{r e}$ tends to 1 exponentially fast as the structure's size $\lambda$ gets small, which is at the heart of the challenge we are addressing in this contribution. In Figure 1(b) we plotted this reflow factor with respect to the targeted periodicity of the structure at the fiber level for a scaling factor $f_{s c}=$ 20. We used a simple semi-empirical model to extract the velocity field $v(z)$ in the preform-tofiber system (see SI 2). In addition, we estimated the temperature distribution, and hence the viscosity, from experimental measurements during drawing (SI 2). The surface tension of PC was taken to be $35.1 \mathrm{mN} \cdot \mathrm{m}^{-1} 37$. An estimated reflow factor is obtained with an error associated for the most part with the assessment of the temperature profile in the furnace during drawing. In Figure 1(b) we hence plotted the reflow factor including a surrounding dashed region that accounts for the error in this measurement that can be estimated to be $\pm 5{ }^{\circ} \mathrm{C}$. Even with this error, the reflow factor accounts very well for the physics behind the observed experimental results: $f_{r e}$ is indeed estimated to be around 0.8 for the $2 \mu \mathrm{m}$ structure, and around 0.5 for the 10 $\mu \mathrm{m}$ structure as indicated on the graph by the red arrows. These values are associated with a large deformation of the initial shape, which corresponds well to the observed texture evolution shown in the SEM micrographs above the graph in Figure 1(b). 
More importantly, this analysis also reveals the innovative approach we demonstrate to avoid the structure collapse for smaller dimensions $(\lambda)$. There are two tunable parameters that depend on intrinsic materials properties, namely the viscosity and the surface tension. The materials that can be pulled at high viscosity are those with high mechanical strength, which can sustain high tension during the draw, such as PEI. Thermoplastics with low surface tension such as fluorinated polymers $\left(15-20 \mathrm{mN} \cdot \mathrm{m}^{-1}\right.$ at $\left.20^{\circ} \mathrm{C}^{[45]}\right)$, are also less likely to reflow. Even with these extreme cases however, our calculations show (see SI 2) that textures below a few microns could not be maintained even for PEI without severe deformation at the fiber level, corresponding to a reflow factor of above 0.25 . Note that one could also play with external parameters such as drawing at higher speed. This requires however to increase the furnace temperature to avoid raising the stress up to fiber breakage, which in turn can lower significantly viscosity for some polymers and accelerate reflow. Here, we do not study the effect of external parameters for the sake of conciseness, but also because our approach makes it irrelevant as it enables to suppress reflow regardless of material, structure, and drawing parameters.

To alleviate the limitations associated with the drawing of a single patterned material, we exploited the thermal drawing of multi-material preforms. By co-drawing two textured polymers in intimate contact as shown schematically in Figure 1(c), we can drastically reduce the interfacial tension of the textured polymers, which results in a drop of the reflow factor. To realize this scheme, we choose carbon-black loaded polyethylene (referred to as cPE) as the sacrificial material, because it provides two key features: it is compatible with thermal codrawing with a variety of thermoplastics such as PC or PMMA ${ }^{[17]}$. Moreover, the interfacial tension between polyethylene and a variety of thermoplastics is significantly reduced compared to the surface tension of individual polymers ${ }^{37}$ (see SI 3). For example, the surface tension of 
PC, PMMA and polyethylene (PE) at $140^{\circ} \mathrm{C}$ are $35.1 \mathrm{mN} \cdot \mathrm{m}^{-1}, 32.1 \mathrm{mN} \cdot \mathrm{m}^{-1}$ and $28.8 \mathrm{mN} \cdot \mathrm{m}^{-1}$ respectively ${ }^{[45]}$, while the interfacial tension between PC and PE is $4.8 \mathrm{mN} \cdot \mathrm{m}^{-1}{ }^{[46]}$ and between PMMA and PE is $9.7 \mathrm{mN} \cdot \mathrm{m}^{-1}[45]$ at the same temperature.

In the graph of Figure 1(b), we plotted in blue the reflow factor when replacing the surface tension of PC with the interfacial tension of PC and PE, keeping all other parameters the same. We indeed show in SI 4 that any effect on the temperature distribution and hence on the viscosity of the textured polymer due to the cPE layer can be neglected. The reflow factor is drastically reduced for a wide range of feature sizes, and textures down to a few tens of nanometers are associated with a reflow factor below 0.1. $f_{\text {re }}$ also remains close to 0 for the same architectures described above for the single-material ribbons, namely the targeted $10-\mu \mathrm{m}$ and $2-\mu \mathrm{m}$ textures. To verify this prediction from our model, we fabricated preforms with a black (cPE) layer sandwiched by two PC (or PMMA) textured plates, as schematically shown in Figure 1(c). We first fabricated the same $100 \mu \mathrm{m}$ and $40 \mu \mathrm{m}$ patterns on PC plates as previously described and hot pressed a cPE layer between the two plates (see methods). We show in the bottom of Figure 1(b) the SEM micrographs of the resulting pattern after thermal drawing and removing of the cPE layer. The textures were perfectly scaled down and conserved even for the $2 \mu \mathrm{m}$ one, which highlights the predicted effect of the cPE layer on preventing thermal reflow.

Finally, to achieve ribbons with exposed textures, we rely on a third useful property of PE, namely its low miscibility with thermoplastics like PC or PMMA ${ }^{[46]}$. While the adhesion after hot pressing of the preform is good enough to sustain the thermal drawing process, it remains weak enough so that the $\mathrm{PC}$ and $\mathrm{cPE}$ parts can be separated at the fiber level using a straightforward mechanical peeling-off process, as shown schematically in Figure 2(a). Note that the particular structure shown in Figure 1(c) with two PC plates results in two textured PC 
ribbons in a single draw. Our scheme hence enables us to engineer the viscous flow during thermal drawing to obtain extended length of textured ribbons (Figure 2(b)). Combined with a fast mechanical peel-off procedure exemplified by the picture in Figure 2(c), textured ribbons of unprecedented complexity and feature sizes can be achieved with high scalability.

Surface textured ribbons, fibers and micro-cavities

In Figure 2(d) we show the typical SEM micrograph of the cross-section of a PC ribbon with a textured surface. The textures we can achieve range from tens of micrometers down to a few hundreds of nanometers, and are organized in any (periodic, aperiodic etc..) configurations as initially selected in the macroscopic preform. Figure 2(e) shows an example of a ribbon with five square-shape structures on its surface, which are $20 \mu \mathrm{m}, 10 \mu \mathrm{m}, 5 \mu \mathrm{m}, 2 \mu \mathrm{m}$ and $1 \mu \mathrm{m}$ in width and depth, from the left respectively, while in Figure 2(f) we show a fiber with a periodic texture reaching a feature size down to $300 \mathrm{~nm}$. We also combined the milling and hot-embossing techniques to create hierarchical structures (Figure 2(g)) with a first texture of $20 \mu \mathrm{m}$ on top of which a second texture of $5 \mu \mathrm{m}$ was fabricated. This is reproduced uniformly over hundreds-ofmeters of fiber length, highlighting the simplicity of our approach to fabricate hierarchical architectures that are difficult to realize with traditional lithographic methods.

Our approach can be equally applied to any fiber geometry including the more conventional circular fibers. To create a multi-material circular preform as shown in Figure 3(a), softembossing was employed in order to transfer the desired pattern onto a polymer film, which was subsequently rolled around a rod made out of the same polymer. The exposed texture was then surrounded by a $\mathrm{cPE}$ film covering the whole pattern before thermal drawing. In Figure 3(a) and 
(b) we show an optical micrograph of a PMMA fiber with an outside texture of $5 \mu \mathrm{m}$ feature size, and a SEM micrograph of a hollow core fiber with a textured inner surface with an $8 \mu \mathrm{m}$ feature size, respectively. Here again, both textures were exactly maintained during the thermal drawing process thanks to the low interfacial tension between PMMA and cPE. Note that we show textures with such scales so that it could be clearly shown that inner surfaces with high curvature could be textured, but much smaller textures could be achieved and were used below. Additionally, beyond exploiting textured fibers in various applications, we also demonstrate their use as molds to create patterns on flat or large curvature surfaces of other materials. A material of choice to prove this concept is PDMS, a silicone elastomer widely used in microfluidics and bioengineering to realize soft micro-structured objects. In Figure 3(c) we show a schematic of our approach for both flat and circular configurations. A PDMS precursor is first cast over the textured PMMA ribbon or fiber. After PDMS curing, the PMMA fiber is removed either manually pulled from the poorly adhered PDMS or dissolved in acetone. This simple process results in textured PDMS films and more strikingly in three-dimensional (3D) PDMS microchannels with a textured inner surface as shown in Figure 3(c). The inner diameter and pattern geometry can be simply tuned to dimensions impossible to achieve with other existing techniques.

\section{Discussion}

The approach we propose constitutes a novel way to impart large area flexible surfaces with submicrometer scale controlled textures. It is also the first time that circular fibers and hence fabrics can be textured with such a high scalability and precision. In more general terms, it is a unique technique to provide textures onto surfaces with very high curvatures. Patterning micro-channels 
is used in microfluidics for example but at the expense of complicated lithography steps and at large feature sizes. Whether it is directly on the fiber or transferred onto another polymer, arbitrary textures can be imparted to surfaces with any curvature with our approach, at the scalability and cost associated with optical fiber processes. To highlight and discuss the potential of this technique, we present a series of examples of applications where textured fibers and soft channels could be highly relevant.

\section{Tailored hydrophobicity}

Textures have a well-known effect on the hydrophobic properties of surfaces ${ }^{[47]}$. To study this effect on fibers and ribbons, we assembled together PC ribbons with a $5 \mu \mathrm{m}$ texture on one side, and a smooth surface on the other. In Figure 4(a) we show the comparison between water droplets deposited on each side of the same assembly, namely a smooth and a textured surface. This approach enables us to rule out any effect of the roughness induced by non-perfect alignment between the fibers, as it is the same in both configurations. Using an OCA 34 DataPhysics Instruments with distilled water drops, we could extract a contact angle of $78^{\circ}$ for the smooth side, and of $105^{\circ}$ for textured surface fibers. Fibers and fabrics with controlled corrugated textures extending along their entire length could hence exhibit interesting and tunable hydrophobic properties.

\section{Optical gratings}

To highlight the sub-micrometer scale of the achieved textures, and the resulting optical effects, we investigated the diffraction pattern generated in transmission by ribbons with various texture sizes. Each ribbon was positioned at the center of a rotating stage and illuminated by a monochromatic beam from a supercontinuum source (Supreme K from NTK Photonics). A 
detector was positioned at the periphery of the stage and measured the optical intensity in the far field at every degree between $-60^{\circ}$ and $60^{\circ}$. This enabled us to measure the angular distribution of the diffracted pattern and extract the angle for the first order peak. In the graph of Figure 4(b) we plotted the measured first order angles versus the texture size for four different textures at an illuminating wavelength of $600 \mathrm{~nm}$. In red we show the simulated value obtained from a FDTD code (Lumerical solution) that agree very well with our experiment. This study reveals that the textures down to $500 \mathrm{~nm}$ are well maintained and exhibit the expected optical properties. On the right in Figure 4(b) we show the reflection of an impinging incandescent light beam on a fiber array with a $500 \mathrm{~nm}$ texture. The left and the middle images were taken on the same fiber array with a different angle of illumination, while the right image shows the reflection on a woven array. This highlights the potential of sub-micrometer textured fibers and ribbons to tailor optical properties of large-area and flexible surfaces and textiles.

\section{Cellular alignment on textured surfaces}

Cell behavior can also be controlled by substrate topography ${ }^{[48]}$. To date, photolithographic techniques borrowed from the microelectronics industry allowed for the fabrication of patterned surfaces. Using patterned PC fibers, we prepared a range of surfaces with parallel square grooves of similar pattern as in Figure 2(a). All substrates were coated with a solution of fibronectin to ensure robust cell attachment and proliferation. Four texture sizes were considered: $10 \mu \mathrm{m}, 5 \mu \mathrm{m}, 1 \mu \mathrm{m}$, and $0.5 \mu \mathrm{m}$; in addition, a flat PC fiber was used as reference substrate. As shown in Figure 5, the cell behavior was remarkably affected by the surface patterns. On the control flat substrates, the cells adhere to the surface through several focal adhesion points distributed homogenously across the cell, without any preferential orientation (Figure 5(a)). 
Grooved patterns in the range of 5-10 $\mu \mathrm{m}$ resulted in alignment of the cells along the pattern direction [Figure 5b,5d], with the cell nuclei elongating into the groove (see SI 6). Small pattern-size substrates $(0.5 \mu \mathrm{m}$ and $1 \mu \mathrm{m}$ grooves $)$ induced an alignment of the cell features, such as focal adhesion and actin fibers [Figure 5(c)] as the cells were able to stretch along the pattern direction. Moreover, a disparate characteristic in terms of cell area was observed: cells grown on large-groove arrays (5 $\mu \mathrm{m}$ and $10 \mu \mathrm{m}$ width) were significantly smaller than cells grown on narrow-groove arrays $(* * * \mathrm{p}<0.001)$, and displayed a more homogeneous size distribution (Figure 5e-f).

We next cultured dorsal root ganglia (DRG) explants for up to 6 days on sheets of textured PDMS membranes. As a control, in Figure 6a we show the neurites sprouting randomly in all directions from dorsal rout on a flat glass substrate. The PDMS membranes were first molded against parallel PC fibers with a groove dimension of $0.5 \mu \mathrm{m}$ [Figure 6b] and $5 \mu \mathrm{m}$ (Figure 6c) bonded together to form a large surface area of several $\mathrm{cm}^{2}$. As expected, neurites sprouting from dorsal root aligned along the grooves, independently on their depth and pitch. DRG explants could also be cultured within the hollow core of a PC fiber with a $5 \mu \mathrm{m}$ textured inner surface (Figure 6d). We used PC for its excellent transparency in the visible enabling to image neurites sprouting through the cladding. A DRG was seeded at the entrance of the cylinder and we monitored neurites sprouting directly within the tube. Neurites grew orderly within the fiber and along the grooves.

The effect of patterned substrate on cell behavior has been previously reported ${ }^{[38],[49]}$ but our drawing process allows the production of substrates and tubes textured with high reproducibility and resolution, over extended length of fibers and ribbons. We can also reach small feature sizes down to sub-micrometer that could be associated with beneficial effects on cell growth and 
orientation. This supports the requirement for small groove sizes for beneficial effect on regenerative scaffold ${ }^{[44]}$. Furthermore, our process may be extended to a wide range of polymers, from transparent PC or PMMA to stretchable and even bio-degradable materials. This range of properties can be exploited to better image the effect of texture on cell growth, to study the effect of the substrates' mechanical properties, or to exploit biodegradability during a regenerative process for nutrients delivery or self-degradation of a scaffold. We therefore anticipate that this technique will offer unique opportunities in bio- and neural engineering, both in the context of in vitro studies and in vivo regenerative strategies.

\section{Conclusion}

In conclusion, we have reported the straightforward and scalable fabrication of sub-micron structures on 2D surfaces or 3D structures such as hollow channels, made of different polymer fibers and ribbons using the preform-to-fiber thermal drawing technique. We combined for the first time soft lithography approaches at the preform level to create patterns at the tens of micrometers scale, with the thermal drawing process to scale down these initial patterns into submicrometer corrugated textures that extend over kilometers of fiber length. To overcome the thermal reflow responsible for the collapse of smaller scale patterns, we covered the structured polymer by a sacrificial layer in order to drastically reduce the interfacial tension that drives the reflow. Patterns down to a few hundreds of nanometers feature size were for the first time obtained on ribbons and fibers, two orders of magnitude below what has been previously demonstrated in literature. To highlight the effect and opportunities of our approach, textured fibers were deployed to create enhanced hydrophobicity surfaces, to make fiber assemblies with optical effects, or used as templates for the growth of biological cells where a clear effect of the 
periodic pattern was observed in their alignment and growth. Textured fibers were also used as molds to fabricate PDMS constructs with hollow channels having textures on their inner surfaces. This represents an original and unique approach to fabricate sub-micrometer textures onto surfaces with very high curvatures for which current patterning techniques are not adapted. Such patterned micro-channels can be used in a myriad of applications in the physical and life sciences. We have shown how the neurites from a DRG explants could grow orderly, paving the way towards novel nerves or neurons regeneration scaffolds, bio-sensing and tissue engineering opportunities. Other applications can be envisioned for example in microfluidics, where channels with textures can mold the flow of liquid or nanoparticles in novel ways. New fiber probes with advanced microfluidics architectures can also impact current diagnosis and treatment strategies. Our approach hence enables the manufacturing of complex sub-micrometer textures within unconventional fibers, ribbons and micro-channels, at the simplicity, scalability and cost traditionally associated with optical fibers.

\section{Methods:}

Preform fabrication and thermal drawing

The Silicon masks were fabricated using Photolithography process at the Centre for MicroNanofabrication (CMi) at EPFL. The initial patterns were created by a Heidelberg DWL200 laser writer on Cr-blank masks, then transferred to Silicon masks by a Suss MA6 mask aligner. The developed Silicon masks were subsequently etched using a plasma etcher Alcatel AMS 200 SE to obtain the desired depth of the pattern. In this work, the etching depth was chosen to be the same as the width of the structure, or half of the period, to have a square shape patterns. This Si mask was then molded onto a PDMS solution via casting (PDMS 84 Dow-Coring) and curing at $80^{\circ} \mathrm{C}$ to transfer the pattern nto a soft PDMS substrate. 
For flat preforms resulting in ribbons after drawing, the predefined patterns on PDMS molds were transferred to PC (Makrolon $\left.{ }^{\circledR}\right)$ and PMMA (Plexiglass $\left.{ }^{\circledR}\right)$. All commercially acquired polymers were first cleaned with isopropanol and stored in vacuum oven for several weeks for degassing before being processed. Plates of PC and PMMA were then hot pressed against the PDMS mask at $175^{\circ} \mathrm{C}$ and $145^{\circ} \mathrm{C}$ respectively accounting for the different glass transition temperatures, at a pressure of $0.1 \mathrm{MPa}$ for 5 minutes using a Thermal NanoImprinter EHN-3250. The multi-material flat preforms with the cPE sacrificial layer were fabricated by sandwiching a cPE (acquired from Goodfellow, UK ) layer with two textured plates, and hot pressing the whole assembly at a pressure of $0.1 \mathrm{MPa}$ for 5 minutes at temperature of $150^{\circ} \mathrm{C}$ and $120^{\circ} \mathrm{C}$ for $\mathrm{PC}$ and PMMA respectively.

Circular preforms were fabricated by the rolling and consolidation technique and annealed in a vacuum oven for consolidation (at $150^{\circ} \mathrm{C}$ for PMMA, and $190^{\circ} \mathrm{C}$ for PC for about 10 minutes). The last rolled thinfilm is previously textured using an approach similar to the one for plates described above. A layer of cPE is rolled around this last layer prior to consolidation.

The multimaterial preforms were subsequently drawn using a custom designed draw tower following conventional protocols ${ }^{25}$. Standard drawing temperatures were set at $265^{\circ} \mathrm{C}$ for PC, and $245^{\circ} \mathrm{C}$ for PMMA.

\section{Scanning Electron Microscopy}

All Scanning Electron Microscopy (SEM) samples were coated with $10 \mathrm{~nm}$ carbon film before transfer into the vacuum chamber. The SEM images were taken with the Zeiss Merlin field emission SEM (Zeiss, Göttingen, Germany) equipped with a GEMINI II column operating between 2.0 and $2.5 \mathrm{kV}$ with a probe current $150 \mathrm{pA}$. The In-Lens annular detector allowed for high resolution secondary electrons imaging at all magnifications.

Optical characterization and modeling 
Optical characterization was performed by placing the fiber at the center of a rotating stage with a fixed source. A monochromatic laser beam (wavelengths $550 \mathrm{~nm}, 600 \mathrm{~nm}$ and $650 \mathrm{~nm}$ ) obtained from a supercontinuum source (Supreme K from NTK Photonics) was incident normally on the sample. A photodetector was positioned at the periphery of the rotating stage to measure the intensity of the diffracted light as a function of angle of diffraction. The detector was rotated from $-60^{\circ}$ to $+60^{\circ}$ to obtain the intensity variation as a function of angle (hence order of diffraction). For each polycarbonate gratings with different feature sizes, the structures were modeled using a commercial programming tool (Lumerical solution). The refractive index of the polycarbonate was taken to be 1.6 in the range of wavelengths considered. Periodic boundary conditions were used in the $\mathrm{x}$ and $\mathrm{z}$ directions (see Figure 1(a), the light being incident along the y direction). A plane wave source was used with a fixed wavelength $(550 \mathrm{~nm}, 600 \mathrm{~nm}$ and $650 \mathrm{~nm})$ and a 0 polarization angle. A built-in grating transmission analyzer of the software was used to calculate the variation of the angle of the first order diffraction with the grating feature size for each wavelength.

\section{Biological samples preparation and characterization}

For experiments with stem cells and DRG, fibers with patterns ranging between $500 \mathrm{~nm}$ and $10 \mu \mathrm{m}$ were selected. Prior to cell culture, the fibers were soaked overnight in ethanol (70\%) and dried in aseptic conditions under a biological cabinet.

\section{Stem Cells}

Adipose-derived stem cells (ASC) were isolated from rats using a previously established protocol [1], and in accordance with the local veterinary commission of the canton of Vaud (Lausanne, Switzerland). Following euthanasia, visceral and inguinal fat was harvested, digested with a collagenase type I solution (Gibco) $(0.2 \% \mathrm{w} / \mathrm{v})$, and finally filtrated through a $100 \mu \mathrm{m}$ cell strainer. The remaining cells were then incubated at $37{ }^{\circ} \mathrm{C}, 5 \% \mathrm{CO}_{2}$ in standard culture flasks sub-cultured in growth medium (minimum essential medium ( $\alpha$-MEM, Gibco), foetal bovine serum (10\%), and penicillin-streptomycin( $1 \%)$ ). 
The fiber substrates were preliminary coated with a solution of fibronectin with a final concentration of 2 $\mu \mathrm{g} / \mathrm{cm}^{2}$. To assess cell response as function of the patterns' size, ASC were seeded on each fiber substrate at a density of 3,000 cells $/ \mathrm{cm}^{2}$ and incubated at $37{ }^{\circ} \mathrm{C}, 5 \% \mathrm{CO}_{2}$ for 3 days. Cells were fixed after 24 and 72 hours in formalin (10\%) and stained to analyze their morphology. Briefly, cells were permeabilized with Triton-X $(0.1 \%)$ /Tween $(0.1 \%)$ in PBS, and non-specific antigens blocked in bovine serum albumin (BSA) $(1 \% \mathrm{w} / \mathrm{v})$ for 30 minutes at room temperature. Samples were incubated in monoclonal anti-vinculin antibody (Sigma; $1: 400$ ) overnight at $4{ }^{\circ} \mathrm{C}$, then the samples were washed again with PBS and incubated with the secondary antibody (AlexaFluor594 goat anti-mouse, LifeTechnologies; 1:1000) for 1 hour in the dark. Phalloidin (AlexaFluor488, LifeTechnologies; 1:40) was finally added for 20 minutes at room temperature to stain cytoskeletal structures. Cells were then imaged using a fluorescence microscope (Leica DMI3000B).

Samples fixed after 24 hours were used for further morphological characterization $(n=3)$. Briefly, images were processed using ImageJ [2] and around 100-180 cells per condition were analyzed for cell area, aspect ratio, Feret's diameter, nuclear area and aspect ratio, including cell and nuclear orientation. Data were processed with Prism 5.03 (GraphPad) and OriginPro 8.6.0 (OriginLab Corporation).

\section{Dorsal Root Ganglia (DRG)}

Following rat euthanasia, the spinal column was carefully removed and the dorsal root ganglia (DRG) roots exposed [1]. DRG were then pulled, cleaned and cultured on laminin-coated fibers and control flat glass slides using Dulbecco's Modified Eagle Medium/Nutrient Mixture F-12 (DMEM/F-12, 1:1, GlutaMAX, LifeTechnologies) medium supplemented with $50 \mathrm{ng} \cdot \mathrm{ml}^{-1}$ of nerve growth factor (NGF 7.5S, LifeTechnologies). The explants were incubated at $37^{\circ} \mathrm{C}, 5 \% \mathrm{CO}_{2}$, changing the medium every 2-3 days.

After 6 days, the samples were fixed in $10 \%$ formalin solution for 40 minutes, permeabilized and stained overnight at $4^{\circ} \mathrm{C}$ against mouse anti-S100 and rabbit $\beta$-Tubulin (Abcam, UK; 1:100 and 1:500 respectively). AlexaFluor594 goat anti-mouse and AlexaFluor488 donkey anti-rabbit were conjugated to 
each relative primary antibody, and the cells nuclei stained with DAPI solution $\left(0.6 \mu \mathrm{g} \cdot \mathrm{ml}^{-1}\right)$. Tissues were finally imaged at different magnifications using an upright confocal microscopy (Zeiss LSM 700).

\section{Acknowledgements:}

The authors are very grateful to the staff of EPFL Centre for Micro-Nanofabrication (CMi), the Centre for electron Microscopy (CIME) and of the ATMX (EPFL-IMX) for their help in making preforms. We also would like to acknowledge the Swiss National Science foundation (grant CR32I2_149609 and grant 200021_146871), the Swiss Competence Center for Materials Science and Technology (CCMX) challenge funding scheme, the European Research Council (ERC Starting Grant 679211 "FLOWTOINCS") and the Bertarelli Foundation for their funding support.

\section{References}

[1] M. Alexander Schmidt, A. Argyros, F. Sorin, Adv. Opt. Mater. 2016, 4, 13.

[2] A. F. Abouraddy, M. Bayindir, G. Benoit, Nat. Mater. 2007, 6.

[3] G. Tao, A. M. Stolyarov, A. F. Abouraddy, Int. J. Appl. Glas. Sci. 2012, 3, 349.

[4] W. Zeng, L. Shu, Q. Li, S. Chen, F. Wang, X. M. Tao, Adv. Mater. 2014, 1.

[5] G. Kostovski, P. R. Stoddart, A. Mitchell, The optical fiber tip: An inherently lightcoupled microscopic platform for micro- and nanotechnologies. Adv. Mater. 2014, 26, 3798-3820.

[6] N. Yu, F. Capasso, J. Light. Technol. 2015, 33, 2344.

[7] M. Consales, M. Pisco, A. Cusano, Photonic Sensors 2012, 2, 289.

[8] B. O’Connor, K. P. Pipe, M. Shtein, Appl. Phys. Lett. 2008, 92, 2006.

[9] R. He, P. J. a. Sazio, A. C. Peacock, N. Healy, J. R. Sparks, M. Krishnamurthi, V. Gopalan, J. V. Badding, Nat. Photonics 2012, 6, 174.

[10] R. He, T. D. Day, M. Krishnamurthi, J. R. Sparks, P. J. a Sazio, V. Gopalan, J. V Badding, Adv. Mater. 2013, 25, 1461.

[11] P. J. A. Sazio, Science (80-. ). 2006, 311, 1583.

[12] H. K. Tyagi, M. A. Schmidt, L. Prill Sempere, P. S. Russell, Opt. Express 2008, 16, 
17227.

[13] S. Wang, C. Jain, L. Wondraczek, K. Wondraczek, J. Kobelke, J. Troles, C. Caillaud, M. a. Schmidt, Appl. Phys. Lett. 2015, 106, 201908.

[14] P. Russell, Science 2003, 299, 358.

[15] A. Argyros, J. Light. Technol. 2009, 27, 1571.

[16] S. D. Hart, G. R. Maskaly, B. Temelkuran, P. H. Prideaux, J. D. Joannopoulos, Y. Fink, Science 2002, 296, 510.

[17] A. M. Stolyarov, L. Wei, F. Sorin, G. Lestoquoy, J. D. Joannopoulos, Y. Fink, Appl. Phys. Lett. 2012, 101, 11108.

[18] A. M. Stolyarov, L. Wei, O. Shapira, F. Sorin, S. L. Chua, J. D. Joannopoulos, Y. Fink, Microfluidic directional emission control of an azimuthally polarized radial fibre laser. Nat. Photonics 2012, 6, 229-233.

[19] J. Ballato, T. Hawkins, P. Foy, R. Stolen, B. Kokuoz, M. Ellison, C. McMillen, J. Reppert, a M. Rao, M. Daw, S. R. Sharma, R. Shori, O. Stafsudd, R. R. Rice, D. R. Powers, Opt. Express 2008, 16, 18675.

[20] A. F. Abouraddy, O. Shapira, M. Bayindir, J. Arnold, F. Sorin, D. S. Hinczewski, J. D. Joannopoulos, Y. Fink, Nat. Mater. 2006, 5, 532.

[21] F. Sorin, O. Shapira, A. F. Abouraddy, M. Spencer, N. D. Orf, J. D. Joannopoulos, Y. Fink, Nano Lett. 2009, 9, 2630.

[22] A. Tuniz, K. J. Kaltenecker, B. M. Fischer, M. Walther, S. C. Fleming, A. Argyros, B. T. Kuhlmey, Nat. Commun. 2013, 4, 2706.

[23] M. Bayindir, F. Sorin, A. Abouraddy, J. Viens, Nature 2004, 431.

[24] F. Sorin, G. Lestoquoy, S. Danto, J. D. Joannopoulos, Y. Fink, Opt. Express 2010, 18, 24264.

[25] F. Sorin, A. F. Abouraddy, N. Orf, O. Shapira, J. Viens, J. Arnold, J. D. Joannopoulos, Y. Fink, Adv. Mater. 2007, 19, 3872.

[26] J. Ballato, T. Hawkins, P. Foy, C. McMillen, L. Burka, J. Reppert, R. Podila, a M. Rao, R. R. Rice, Opt. Express 2010, 18, 4972.

[27] A. Gumennik, A. M. Stolyarov, B. R. Schell, C. Hou, G. Lestoquoy, F. Sorin, W. McDaniel, A. Rose, J. D. Joannopoulos, Y. Fink, Adv. Mater. 2012, 24, 6005.

[28] A. M. Stolyarov, A. Gumennik, W. McDaniel, O. Shapira, B. Schell, F. Sorin, K. Kuriki, G. Benoit, A. Rose, J. D. Joannopoulos, Y. Fink, Opt. Express 2012, 20, 12407.

[29] N. D. Orf, O. Shapira, F. Sorin, S. Danto, M. A. Baldo, J. D. Joannopoulos, Y. Fink, Proc. Natl. Acad. Sci. U. S. A. 2011, 108, 4743. 
[30] E. Banaei, A. F. Abouraddy, 2015, 403.

[31] A. Canales, X. Jia, U. P. Froriep, R. a Koppes, C. M. Tringides, J. Selvidge, C. Lu, C. Hou, L. Wei, Y. Fink, P. Anikeeva, Nat. Biotechnol. 2015, 2, 1.

[32] C. Lu, U. P. Froriep, R. a. Koppes, A. Canales, V. Caggiano, J. Selvidge, E. Bizzi, P. Anikeeva, Adv. Funct. Mater. 2014, 24, 6594.

[33] M. Bayindir, O. Shapira, D. Saygin-Hinczewski, J. Viens, A. F. Abouraddy, J. D. Joannopoulos, Y. Fink, Nat. Mater. 2005, 4, 820.

[34] M. Bayindir, A. F. Abouraddy, J. Arnold, J. D. Joannopoulos, Y. Fink, Adv. Mater. 2006, 18,845 .

[35] M. L. Brongersma, Y. Cui, S. Fan, Nat. Mater. 2014, 13, 451.

[36] I. You, T. G. Lee, Y. S. Nam, H. Lee, ACS Nano 2014.

[37] J. Atencia, D. J. Beebe, Nature 2005, 437, 648.

[38] I. Tonazzini, E. Jacchetti, S. Meucci, F. Beltram, M. Cecchini, Adv. Healthc. Mater. 2015, 4, 1849.

[39] I. I. Stoyanova, R. J. A. Van Wezel, W. L. C. Rutten, J. Neural Eng. 2013, 10, 66018.

[40] M. Sun, M. McGowan, P. J. Kingham, G. Terenghi, S. Downes, J. Mater. Sci. Mater. Med. 2010, 21, 2765.

[41] M. Hamdorf, D. Johannsmann, J. Chem. Phys. 2000, 112, 4262.

[42] M. Henle, A. Levine, Phys. Rev. E 2007, 75, 21604.

[43] A. Yildirim, M. Yunusa, F. E. Ozturk, M. Kanik, M. Bayindir, Adv. Funct. Mater. 2014, $24,4569$.

[44] R. A. Koppes, S. Park, T. Hood, X. Jia, N. Abdolrahim Poorheravi, A. H. Achyuta, Y. Fink, P. Anikeeva, Biomaterials 2016, 81, 27.

[45] S. Wu, Polymer interface and adhesion; Dekker, New York, 1982.

[46] H. T. Pham, C. J. Carrierre, Polym. Eng. Sci. 1997, 37.

[47] P.-G. de Gennes, F. Brochard-Wyart, D. Quéré, Capillarity and Wetting Phenomena: Drops, Bubbles, Pearls, Waves; 2002.

[48] P. Clark, P. Connolly, a S. Curtis, J. a Dow, C. D. Wilkinson, Development 1987, 99, 439.

[49] M. R. Lee, K. W. Kwon, H. Jung, H. N. Kim, K. Y. Suh, K. Kim, K. S. Kim, Biomaterials 2010, 31, 4360. 
Figure 1: Thermal drawing of textured ribbon: (a) Schematic of a textured thermoplastic preform and its subsequent thermal drawing, the red ring representing the heating furnace. (b) top: SEM micrograph of textured fibers drawn with a scale factor of 20 , with targeted periodicity of $2 \mu \mathrm{m}$ (left, scale bar $2 \mu \mathrm{m}$ ) and $10 \mu \mathrm{m}$ (right, scale bars $20 \mu \mathrm{m}$ ). The graph represents the reflow factor versus the targeted periodicity of the texture at the fiber level (see text). The bottom SEM micrograph represent similar textured ribbons as above but that were thermally drawn in contact with a cPE layer which maintained the texture during the draw. (c) Schematic of the preform (left) and thermal drawing (right) of a multi-material preform with textured thermoplastic sandwiching a cPE layer.

(a)

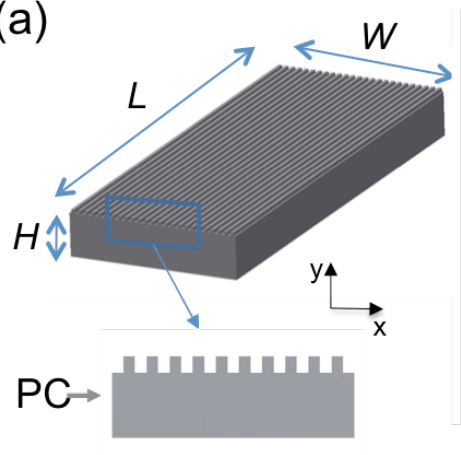

(c)

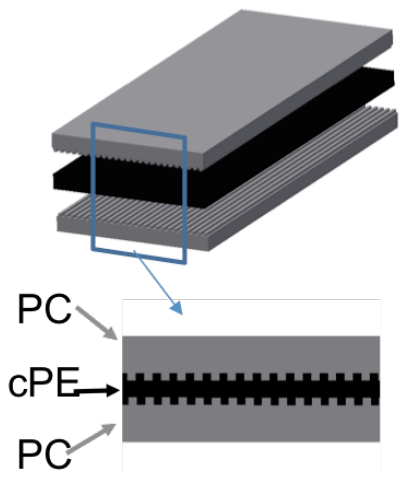

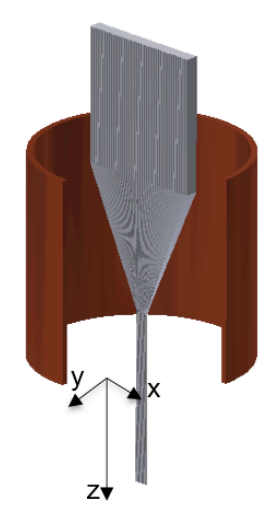

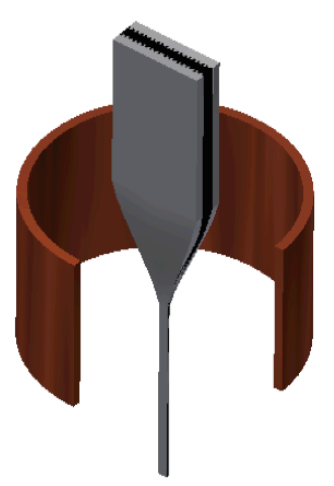

(b)

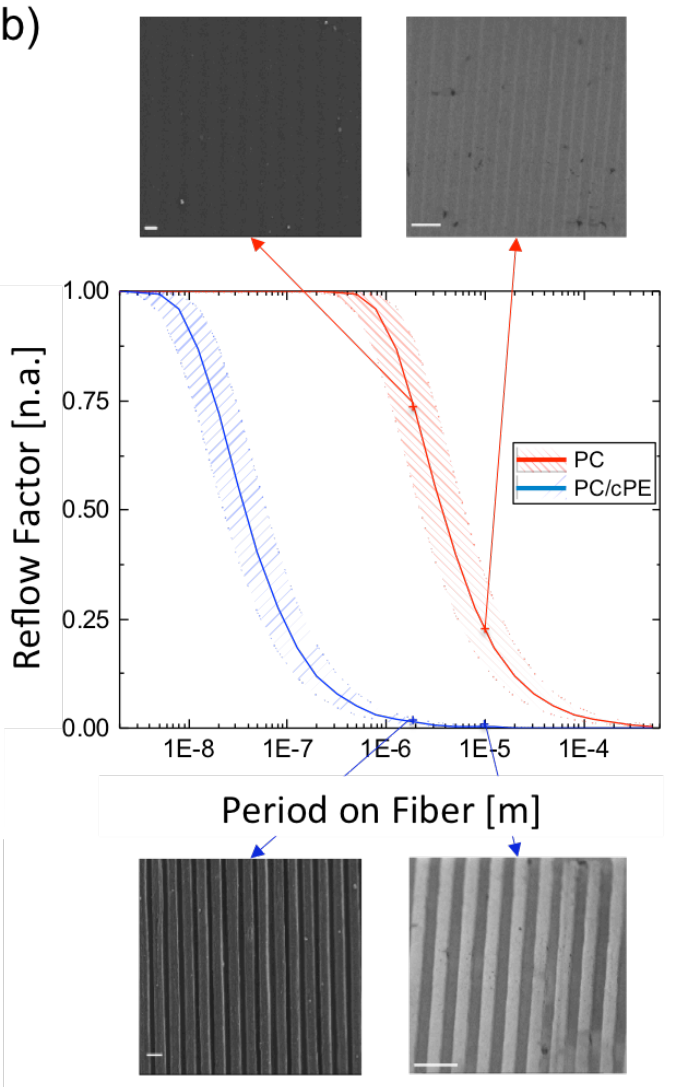


Figure 2 Textured thermoplastic ribbons. (a) Schematic of a preform-to-fiber multi-material ribbon that highlight the separation post-drawing between the different textured layers. (b) Picture of extended length of a thermally drawn multi-material ribbon. (c) Picture of the peelingoff process that separate the textured thermoplastic ribbons from the internal cPE layer. (d) SEM micrograph of a textured ribbon, with an inset of the textured region (scale bar $15 \mu \mathrm{m}$ ). (e) SEM micrograph of the top view of a textured fibers with textures of different height and width (scale bar $10 \mu \mathrm{m}$ ). (f) SEM top view of a square shape textured ribbon with a square shape (scale bar 10 $\mu \mathrm{m})$ with a zoom-in (scale bar $200 \mathrm{~nm}$ ). (g) Schematic of a double-texture ribbon and SEM micrograph of such a double textured ribbon (scale bar $100 \mu \mathrm{m}$ ) with two insets on the first texture (top right, scale bar $20 \mu \mathrm{m}$ ) and the small texture on top of the first one (bottom right, scale bar $1 \mu \mathrm{m})$.

(a)

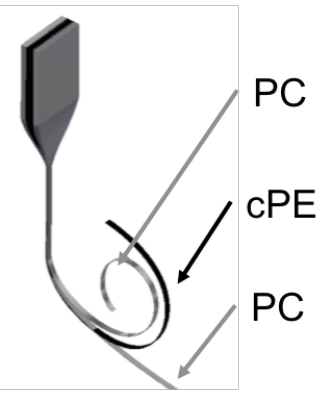

(d)

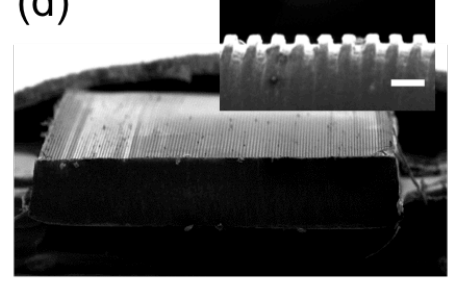

(g)

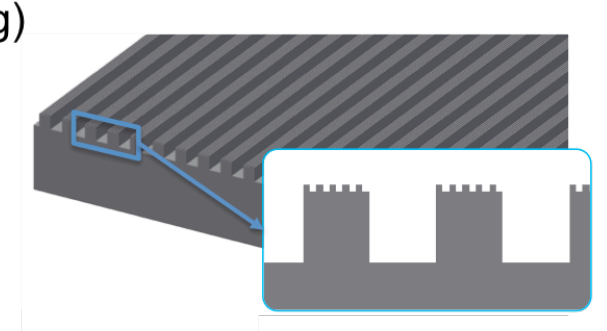

(b)
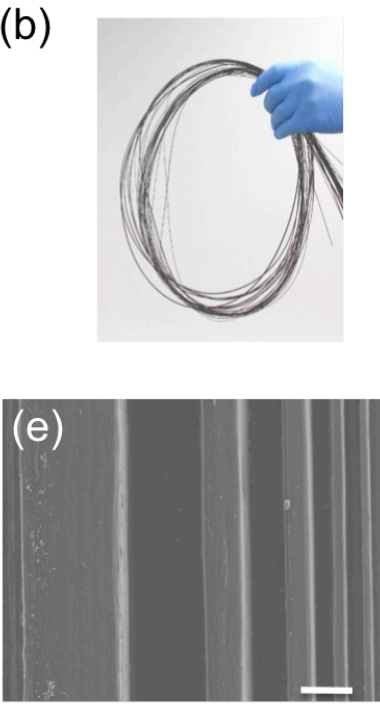

(c)
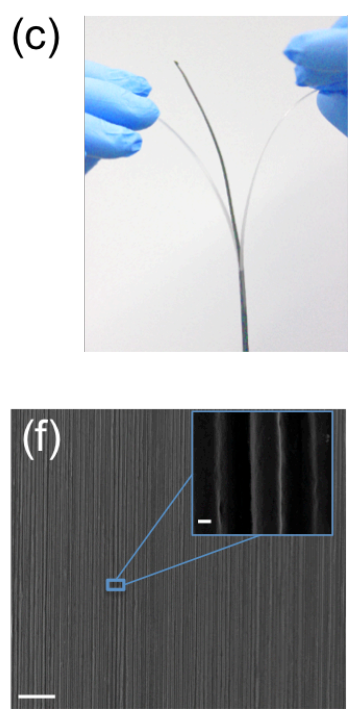

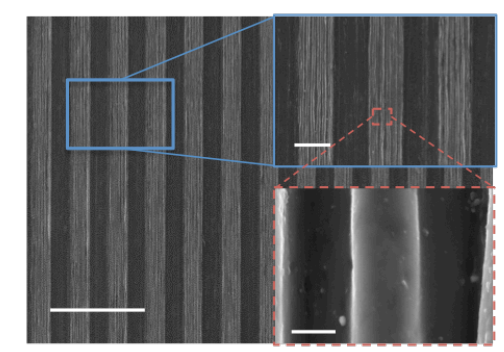


Figure 3: Texture on circular fibers and within hollow channels: (a) Schematic of the thermal drawing of a thermoplastic textured fiber (grey) surrounded by a CPE sacrificial layer in black. An optical micrograph is shown with a scale bar of $20 \mu \mathrm{m}$. (b): SEM image of a hollowcore fiber with internal surface patterned (scale bar top $200 \mu \mathrm{m}$ and bottom $50 \mu \mathrm{m}$ ). (c) Schematic of the approach to realize textured PDMS flat films and microchannels from textured fibers and ribbons molds. (d) Optical micrograph of a PDMS channel with a textured inner wall (bottom, scale bar $50 \mu \mathrm{m}$ ).

(a)

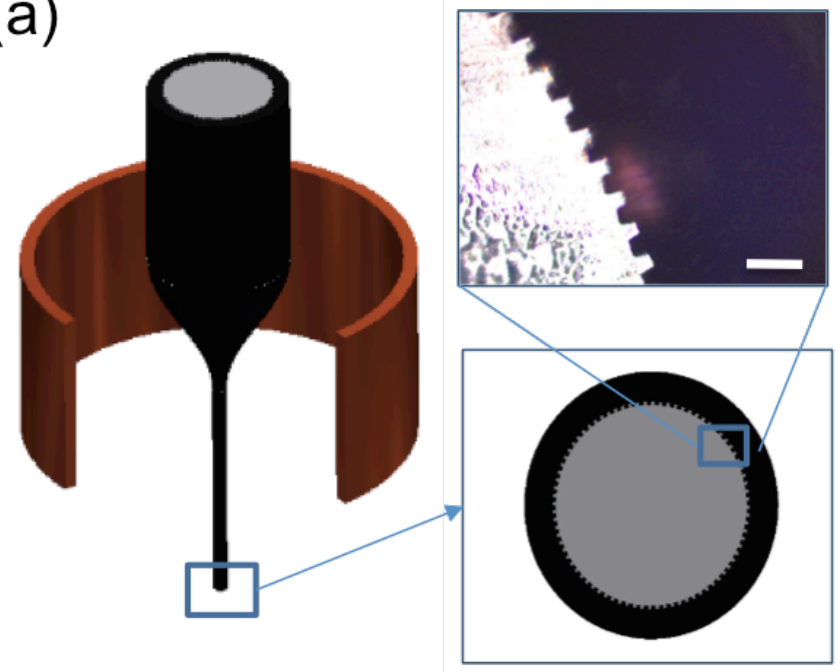

(c)

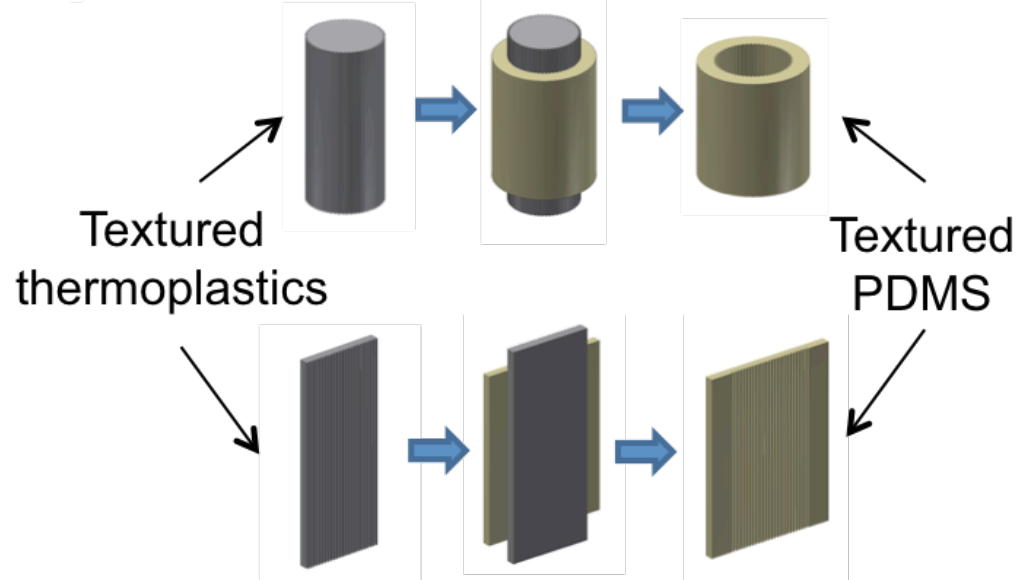

(b)

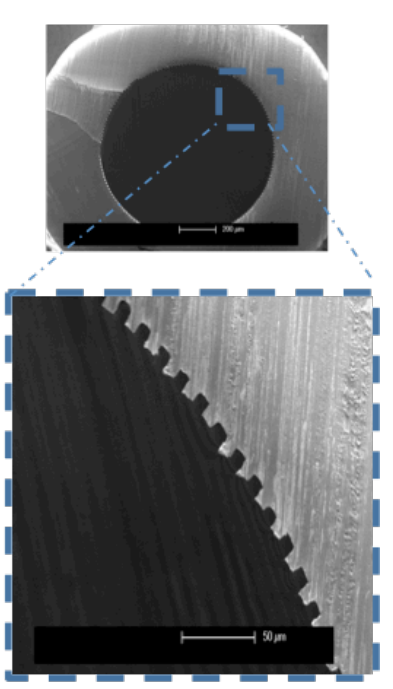

(d)

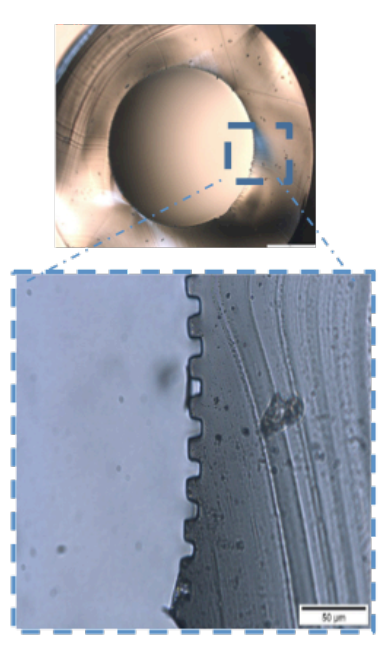


Figure 4: Applications of surface textured fibers. (a) Left: schematics of droplets deposited on a textured fiber assembly, on the smooth and textured parts. The corresponding real micrograph are shown on the right. Contact angles of $78^{\circ}$ (top) and $105^{\circ}$ (bottom) are extracted for dropplets deposited on the smooth and textured size respectively.; (b) Left: graph of the first order diffraction angle versus the squared texture periodicity of a textured ribbon. The points represent experimental data with an error bar associated with the error on the angle measurement and the orientation of the ribbon with respect to the source. The simulated curve is obtained from a carefully designed FDTD calculation using Lumerical. Right: incandescent-light reflection on $0.5 \mu \mathrm{m}$-surface-patterned assembled fibers with different angles (left and middle) and on a woven fiber arrays (right);

(a)
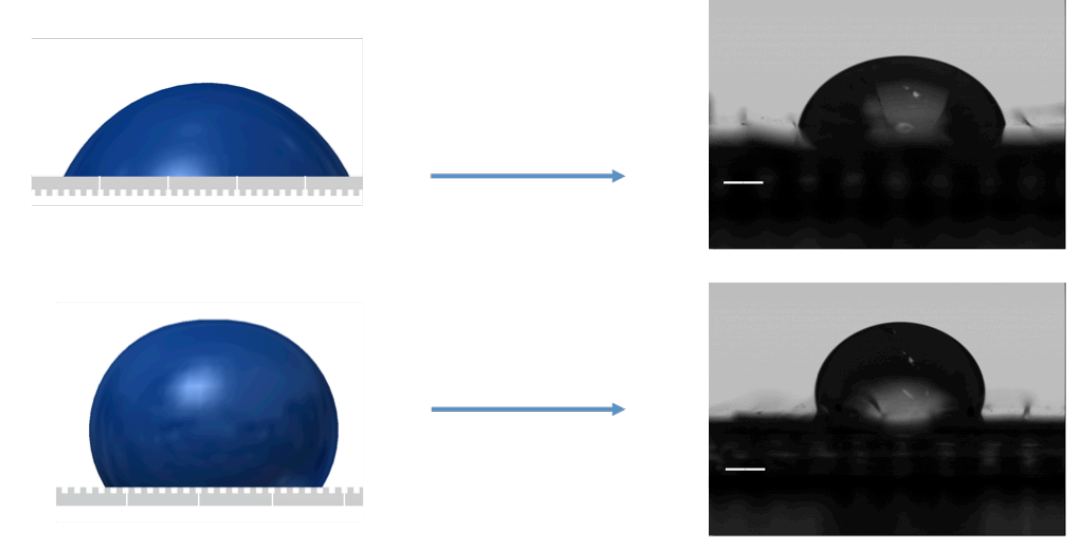

(b)
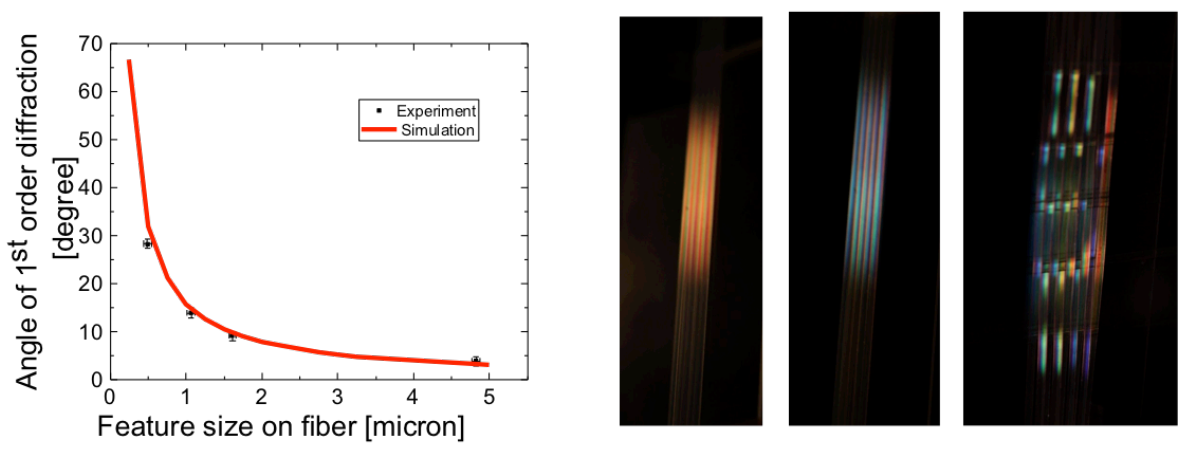
Figure 5: Alignment of adipose derived stem cells on textured fibers surfaces. $(a, b, c)$ left: confocal images of adipose-derived stem cells cultured for 3 days on PC substrates (green $=$ phalloidin, red $=$ focal adhesions, blue $=$ DAPI $),($ scale bar $50 \mu \mathrm{m})$; right: relative orientation frequency of cells, (a) flat fiber, (b) $10 \mu \mathrm{m}$-groove-size patterned fiber, (c) $1 \mu \mathrm{m}$-groove-size patterned fiber. (d) cell division along a $10 \mu \mathrm{m}$-groove-size substrate (scale bar $25 \mu \mathrm{m}$ ). (e) and (f) aspect ratio and cell area on different substrates, ${ }^{* * *} p<0.001$.

(a)

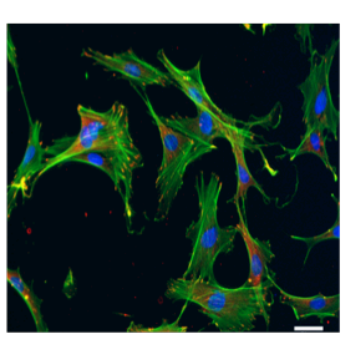

(b)
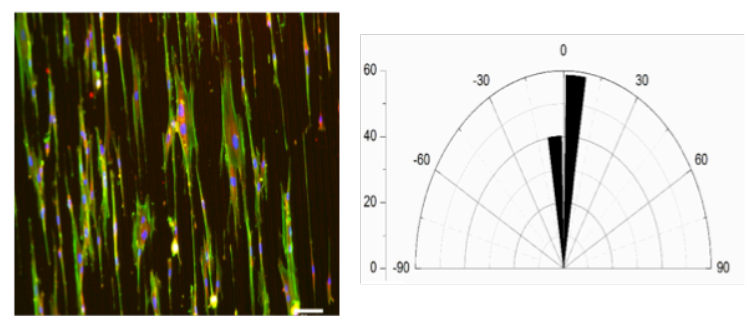

(c)
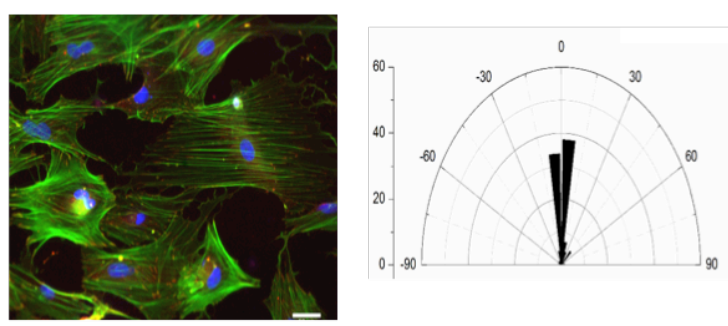

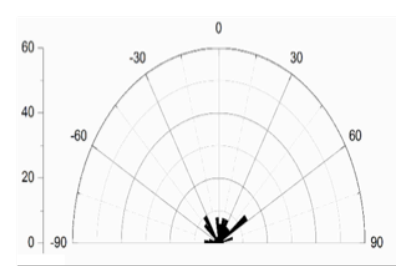

(d)

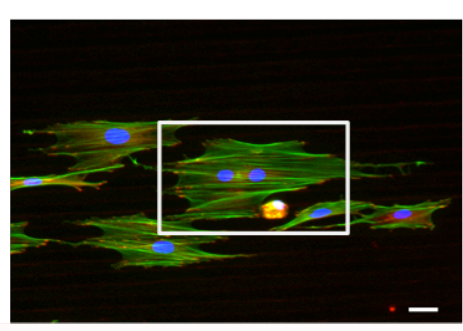

(e)

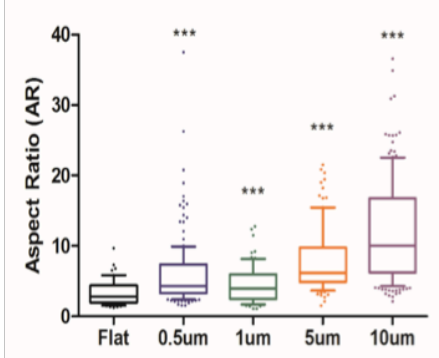

(f)

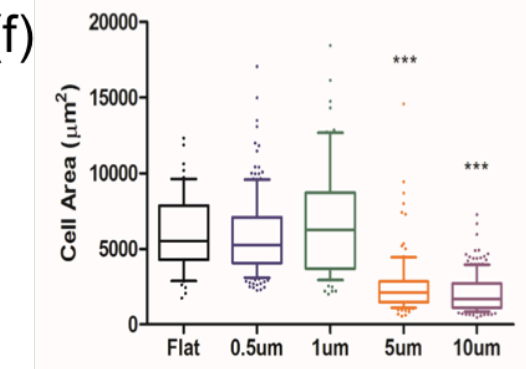


Figure 6: Growth of dorsal root ganglions (DRG) on flat and textured substrates. (a - c) confocal images of DRG explants on (a) a glass slide and (b-c) PDMS patterned substrates casted on thermally drawn patterned ribbons (green $=\beta$-tubulin), scale bar $100 \mu \mathrm{m}$; (b) $0.5 \mu \mathrm{m}$ groove-size pattern, (c) $5 \mu \mathrm{m}$-groove-size pattern; (d) left: schematic of DRG explants growing through a textured hollow conduit ( $1 \mathrm{~mm}$ diameter); right: DRG growing through a hollow-core PC conduit with internal patterned surface of $5 \mu \mathrm{m}$ (scale bar $100 \mu \mathrm{m}$ ).

(a)

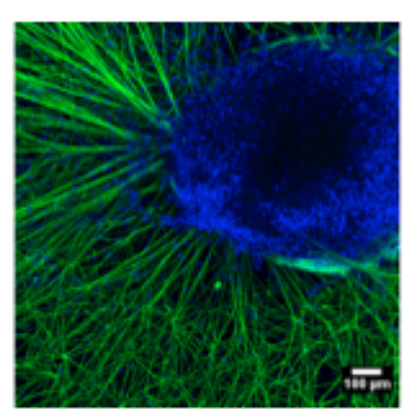

(c)

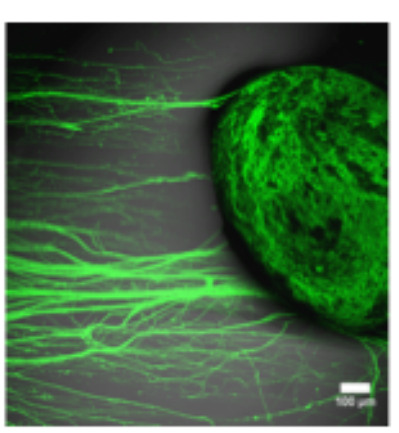

(b)

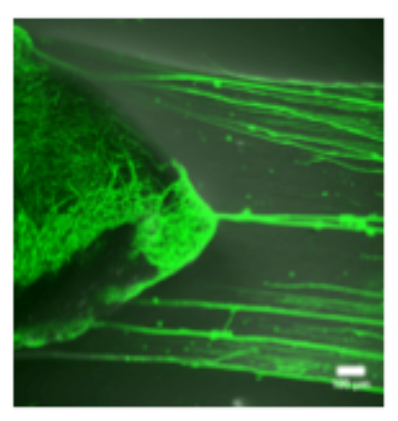

(d)

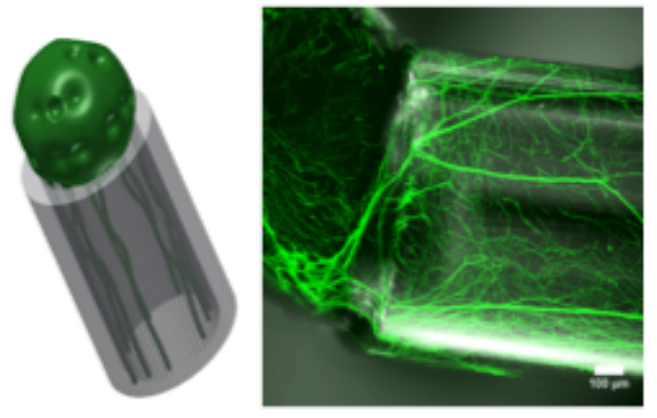

